\title{
Actualización del manejo clínico de COVID-19 en pediatría: a un año de pandemia
}

\author{
Update on clinical management of COVID-19 in \\ pediatric patients: after a year of pandemic
}

\author{
Victoria Eugenia Montaño-Luna,* María Guadalupe Miranda-Novales ${ }^{\ddagger}$ \\ * Departamento de Infectología; ${ }^{\ddagger}$ Unidad de Investigación en Análisis y Síntesis de la Evidencia. Unidad Médica de Alta Especialidad \\ Hospital de Pediatría “Dr. Silvestre Frenk Freund” del Centro Médico Nacional Siglo XXI, Instituto Mexicano del Seguro Social. México.
}

\section{RESUMEN}

En diciembre de 2019 se identificó por primera vez al SARSCoV-2 como la causa de un brote de neumonía grave en China. El 11 de marzo de 2020 la Organización Mundial de la Salud declaró la pandemia por SARS-CoV-2 debido al rápido aumento de casos en el mundo. El periodo de incubación varía de 1-12.5 días (promedio de 5-6 días) y se transmite principalmente por gotas y por contacto directo, siendo menos clara la transmisión por aerosoles en el entorno comunitario. En los niños, la mayoría de los pacientes son asintomáticos o tienen un cuadro respiratorio leve, pero la fuente de contagio casi siempre es intradomiciliaria. Por fortuna, pocos niños llegan a desarrollar manifestaciones graves, como el síndrome multisistémico inflamatorio pediátrico, que consiste en un conjunto de manifestaciones clínicas de hiperinflamación con fiebre prolongada que puede evolucionar a choque y muerte. Lamentablemente, en este momento no hay un tratamiento específico aprobado para su uso en pacientes pediátricos a pesar del tiempo que ha pasado y de que se han realizado múltiples estudios de investigación. Por esta razón, el manejo debe seguir basándose en el tratamiento de soporte y la identificación oportuna de casos. El presente documento tiene como objetivo guiar el manejo de los pacientes pediátricos sospechosos o confirmados de COVID-19 de acuerdo con los hallazgos de estudios de investigación realizados hasta enero de 2021 .

Palabras clave: COVID-19, pandemia, pediatría, guía clínica, tratamiento.

\begin{abstract}
On December 2019, a new virus was announced as the cause of an outbreak of severe pneumonia in China. WHO declared the SARS-CoV-2 pandemic due to the rising cases worldwide On March $11^{\text {th }}$ 2020. The incubation period ranges from 1-12.5 days (average 5-6 days) and its transmission is by droplets, and direct contact, with an unclear transmission during aerosol-generating procedures in the community setting. In almost all pediatric cases, a household contact is the source of infection, and most of these patients are asymptomatic and have a mild respiratory condition. However, few patients develop severe manifestations such as pediatric inflammatory multisystem syndrome temporally associated with SARS-CoV-2 (PIMS-TS) which consists in a wide spectrum of clinical manifestations with hyperinflammatory symptoms that include prolonged fever, hypovolemia and shock that may lead to death. Unfortunately, there is no specific treatment approved for use in pediatric patients, despite the time that has passed and multiple different research studies have been conducted. For this reason, management must continue to be based on supportive treatment and timely case identification. This document aims to guide the management of pediatric patients suspected or confirmed of COVID-19, according to the findings of research studies conducted through January 2021.
\end{abstract}

Keywords: COVID-19, pandemic, pediatrics, clinical guideline, treatment.

Correspondencia: Dra. María Guadalupe Miranda-Novales, guadalupe.mirandan@imss.gob.mx

Citar como: Montaño-Luna VE, Miranda-Novales MG. Actualización del manejo clínico de COVID-19 en pediatría: a un año de pandemia. Rev Mex Pediatr. 2021; 88(1): 31-45. https://dx.doi.org/10.35366/99417 


\section{INTRODUCCIÓN}

En diciembre de 2019 se identificó por primera vez un brote de neumonía de etiología desconocida en un mercado de mariscos y animales exóticos en la ciudad de Wuhan, China. El 9 de enero de 2020 se anunció que la causa era un coronavirus, que inicialmente fue llamado nuevo coronavirus-2019 y que finalmente se nombró como SARS-CoV-2 por su homología genética con SARS-CoV. La enfermedad causada por este virus se nombró como COVID-19 (coronavirus disease) el 11 de febrero de 2020. El 11 de marzo la OMS declaró la pandemia por SARS-CoV-2 debido al rápido aumento de casos en el mundo. ${ }^{1}$

Los coronavirus pertenecen a la familia Coronaviridae e infectan principalmente a aves y mamíferos; pueden modificarse debido a diversos eventos genéticos e infectar al humano, con un potencial epidémico-pandémico, como en el caso de las epidemias de síndrome respiratorio agudo grave (SARS-CoV), el síndrome respiratorio de Oriente Medio (MERS-CoV) y actualmente el SARS-CoV-2, el cual pertenece al grupo de los betacoronavirus y su genoma codifica cuatro proteínas estructurales principales; la proteína S (Spike) es la encargada de unir al virus a los receptores de la célula huésped y facilitar su entrada a través de la membrana celular; ${ }^{2,3}$ lo anterior ha sido clave en el desarrollo de las vacunas ya disponibles, además de las múltiples mutaciones identificadas hasta el momento en los genes que codifican esta proteína como la variante B.1.1.7 identificada de manera reciente, cuya transmisibilidad es aparentemente mayor en un $70 \%$ comparada con otras variantes. ${ }^{4}$

El periodo de incubación de SARS-CoV-2 varía de 1-12.5 días (promedio de 5-6 días) y se transmite principalmente por gotas y por contacto directo, siendo menos clara la transmisión por aerosoles en el entorno comunitario que dentro de unidades médicas. La propagación del virus ocurre a través del contacto persona a persona mediante la expulsión de gotas al hablar, toser o estornudar. ${ }^{5}$ Se ha definido como contacto estrecho a aquel con una persona infectada a una distancia menor a 1.5 metros durante un total acumulado de cuando menos 15 minutos, en un periodo de 24 horas. ${ }^{6,7}$ También se ha documentado la transmisión del virus de personas asintomáticas, llamando a este periodo presintomático, y se ha reportado que hasta $50 \%$ de los contagios suceden a través de personas asintomáticas. ${ }^{8,9}$

Hasta el momento, desde el inicio de la pandemia se han confirmado más de 100 millones de casos y se han alcanzado más de 2.5 millones de defunciones en el mundo. ${ }^{10}$ En México, hasta el 15 de enero de 2021 se confirmaron $1,609,735$ casos y 139,022 defunciones por COVID-19. ${ }^{11}$ De acuerdo con la Secretaría de Salud, los casos de niñas, niños y adolescentes en México reportados al 10 de enero de 2021 son de 40,770 casos. $^{12}$

\section{GENERALIDADES DE COVID-19 EN LA EDAD PEDIÁTRICA}

Hasta el momento existe evidencia de que el espectro clínico de COVID-19 en la edad pediátrica difiere de lo observado en adultos, habiéndose reportado casos en todos los grupos de edad, desde la etapa neonatal hasta la adolescencia. Los primeros reportes de países con tasas altas de infección registraron que sólo de $2-4 \%$ fueron niños. ${ }^{13,14}$ La principal fuente de contagio la constituye un contacto domiciliario. ${ }^{15}$

Estudios iniciales demostraron que el curso de la enfermedad era generalmente leve a comparación con los adultos. Cai y colaboradores reportaron la primera serie de 10 casos de pacientes pediátricos hospitalizados en Sanghai. ${ }^{16}$ Dong y su equipo en China describieron a 2,153 pacientes menores de 18 años, de los cuales se confirmaron 728 casos (34\%) con una media de edad de siete años. La mayoría de los casos se clasificaron como leves $(43.1 \%)$ y sólo $5.9 \%(\mathrm{n}=43)$ fueron graves. ${ }^{17} \mathrm{Lu}$ y colaboradores evaluaron a 1,391 pacientes durante un mes, confirmándose SARS-CoV-2 en 171 (12.3\%), la mediana de edad fue de 6.7 años. El síntoma principal fue fiebre en $41.5 \%$, otros signos y síntomas descritos fueron tos y faringe hiperémica; 27 (15.8\%) pacientes se reportaron asintomáticos y sin signos radiológicos de neumonía. ${ }^{18}$ Posteriormente, a la luz de la publicación de múltiples estudios, en una revisión sistemática con 45 estudios, se determinó que los casos de COVID-19 en pacientes pediátricos solamente correspondían de $1-5 \%$, y que la mayoría cursaba con enfermedad leve. ${ }^{19}$

En el caso de los recién nacidos $(\mathrm{RN})$ se ha documentado una posible transmisión perinatal, la cual ha estado en controversia. Un estudio en China mostró viremia materna en $1 \%$. Se ha reportado detección de SARS-CoV-2 por PCR en exudado nasofaríngeo en RN de uno o dos días de vida, así como niveles elevados de IgM; sin embargo, existe la posibilidad de falsos positivos, así como reacciones cruzadas con otros agentes infecciosos de adquisición perinatal. La complicación obstétrica más frecuente identificada hasta este momento es el nacimiento prematuro con las complicaciones neonatales asociadas. ${ }^{20,21}$

A pesar de que, en general, el curso clínico es benigno y sin complicaciones, es posible que se presente 
un cuadro grave denominado síndrome inflamatorio multisistémico pediátrico asociado con SARS-CoV-2 (PIMS), el cual se describirá más adelante en esta publicación.

\section{CUADRO CLÍNICO}

De acuerdo con lo observado en los estudios realizados en niños, las manifestaciones son menos graves comparadas con lo observado en adultos, incluso en pacientes con inmunocompromiso como los niños con cáncer y postrasplantados; ${ }^{22-24}$ sin embargo, algunos pueden evolucionar y requerir cuidados intensivos. Las manifestaciones clínicas reportadas hasta el momento incluyen:

1. Fiebre.

2. Tos habitualmente seca.

3. Fatiga.

4. Rinorrea.

5. Congestión nasal.

6. Cefalea/irritabilidad.

7. Cianosis.

8. Hiporexia/disminución de la ingesta.

9. Hipoactividad.
10. Rechazo al alimento.

11. Diarrea y otros síntomas gastrointestinales (náusea y vómito).

12. Exantema maculopapular generalizado.

\section{DEFINICIÓN DE CASO}

En la evaluación inicial del paciente que potencialmente tenga COVID-19 debe establecerse una definición de caso; la definición operacional de caso sospechoso de enfermedad respiratoria viral de acuerdo con la Dirección General de Epidemiología en México se describe en la Tabla $1 .^{25}$

En caso de que el paciente cumpla con los criterios para caso sospechoso de COVID-19 se deberá realizar un estudio epidemiológico y una prueba diagnóstica. Mientras que con base en el escenario clínico se decidirá el manejo adecuado (Tabla 2).

\section{CONSIDERACIONES ESPECIALES EN PACIENTES PEDIÁTRICOS CON CÁNCER}

Los estudios realizados hasta el momento reportan manifestaciones de COVID-19 de menor gravedad comparadas con las observadas en adultos con cáncer;

Tabla 1: Definiciones de caso (México).

Caso sospechoso

Caso confirmado por laboratorio

Caso confirmado por prueba antigénica rápida para SARS-CoV-2

Definiciones operacionales en recién nacidos

Caso sospechoso: recién nacido asintomático o con enfermedad respiratoria aguda leve o tos que tenga alguna de las siguientes:

1. Hijo de madre con enfermedad respiratoria aguda leve o grave que a su vez sea caso sospechoso desde 14 días antes, al momento del nacimiento o durante los primeros 28 días de vida

2. Haber estado en contacto con cualquier persona de caso sospechoso o confirmado durante los primeros días de vida

Persona de cualquier edad que en los últimos 10 días haya presentado al menos uno de los siguientes signos y síntomas: tos, disnea, fiebre o cefalea*; y al menos uno de los siguientes:

- Disnea (dato de gravedad)

- Artralgias

- Mialgias

- Odinofagia/ardor faríngeo

- Rinorrea

- Dolor torácico

- Anosmia

- Ageusia o disgeusia

- Conjuntivitis

Persona que cumpla con la definición operacional de caso sospechoso y que cuente con diagnóstico confirmado por la Red Nacional de Laboratorios de Salud Pública reconocidos por el InDRE

Persona que cumple con definición operacional de caso sospechoso y cuente con una prueba antigénica rápida positiva para SARS-CoV-2 mediante estuche comercial avalado por el InDRE

InDRE = Instituto Nacional de Diagnóstico y Referencia Epidemiológicos.

${ }^{*}$ En menores de cinco años la irritabilidad sustituye a la cefalea. 
Tabla 2: Escenario clínico y manejo de los casos sospechosos de COVID-19., 5,26,27

\begin{tabular}{|c|c|c|}
\hline Diagnóstico & Criterios diagnósticos & Manejo \\
\hline \multirow[t]{2}{*}{$\begin{array}{l}\text { Infección de vías } \\
\text { aéreas superiores } \\
\text { no complicada }\end{array}$} & $\begin{array}{l}\text { Síntomas inespecíficos como tos, fiebre, dolor faríngeo, } \\
\text { congestión nasal, malestar general, cefalea, dolor muscular. } \\
\text { Sin signos de deshidratación, sepsis o dificultad respiratoria }\end{array}$ & Ambulatorio (excepto grupos de riesgo, ver más adelante) \\
\hline & & $\begin{array}{l}\text { Tratamiento sintomático: paracetamol de } 10-15 \mathrm{mg} / \mathrm{kg} / \mathrm{dosis} \\
\text { cada } 6 \text { a } 8 \text { h en caso de fiebre. Control por medios físicos } \\
\text { Continuar con lactancia materna y alimentación propia para } \\
\text { la edad, abundantes líquidos }\end{array}$ \\
\hline \multirow[t]{6}{*}{ Neumonía } & Tos y taquipnea de acuerdo con la edad & $\begin{array}{l}\text { Hospitalario en área para la atención de pacientes } \\
\text { COVID-19 }\end{array}$ \\
\hline & $<2$ meses: $>60 x^{\prime}$ & Monitorización continua \\
\hline & 2-11 meses: $>50 x^{\prime}$ & $\begin{array}{l}\text { Biometría hemática, proteína } C \text { reactiva, procalcitonina, } \\
\text { hemocultivos en caso de fiebre, transaminasas, función } \\
\text { renal, pruebas de coagulación, dímero D }\end{array}$ \\
\hline & $1-5$ años: $>40 x^{\prime}$ & $\begin{array}{l}\text { Evaluar el inicio de tratamiento antimicrobiano empírico con } \\
\text { resultados de laboratorio y presentación clínica }\end{array}$ \\
\hline & Adolescentes y adultos: $>30 x^{\prime}$ & Cefuroxima 150 mg/kg/día IV \\
\hline & Saturación ambiental: > 92\% & \\
\hline Neumonía grave & $\begin{array}{l}\text { Tos o dificultad respiratoria y al menos uno de los siguientes: } \\
\text { - Cianosis central o } \mathrm{SaO}_{2}<92 \% \\
\text { - Dificultad respiratoria: quejido, aleteo nasal, tiraje supraesternal, } \\
\text { retracción xifoidea o disociación toracoabdominal } \\
\text { - Gasometría arterial: } \\
\text { - } \mathrm{PaO}_{2}<60 \mathrm{mmHg} \\
\text { - } \mathrm{PaCO}_{2}>50 \mathrm{mmHg}\end{array}$ & $\begin{array}{l}\text { Unidad de Cuidados Intensivos (UCI) en área para la } \\
\text { atención de pacientes COVID-19 } \\
\text { Monitorización continua } \\
\mathrm{BH}, \mathrm{PCR}, \mathrm{PCT} \text {, hemocultivos en caso de fiebre, } \\
\text { transaminasas, función renal, coagulación, dímero D } \\
\text { Líquidos IV, oxigenoterapia para mantener } \mathrm{SO}_{2}>92 \% \text {, } \\
\text { evitar procedimientos generadores de aerosoles (Tabla 3) } \\
\text { Evaluar el inicio de tratamiento antimicrobiano empírico } \\
\text { Cefotaxima } 150 \mathrm{mg} / \mathrm{kg} / \mathrm{día}+\text { vancomicina } 60 \mathrm{mg} / \mathrm{kg} / \mathrm{día}\end{array}$ \\
\hline \multirow{2}{*}{$\begin{array}{l}\text { Síndrome de } \\
\text { distrés respiratorio } \\
\text { agudo (SDRA) }\end{array}$} & - Inicio súbito o empeoramiento del cuadro & Considerar el uso de inmunomoduladores \\
\hline & $\begin{array}{l}\text { - Rx tórax, TAC: nuevo(s) infiltrado(s) uni/bilaterales } \\
\text { compatibles con afección aguda del parénquima pulmonar. } \\
\text { Imagen en vidrio despulido } \\
\text { - Edema pulmonar: en ausencia de otra etiología } \\
\text { - Oxigenación }(\mathrm{OI}=\text { índice de oxigenación y } \mathrm{OSI} \text { = índice de } \\
\left.\text { oxigenación usando } \mathrm{SpO}_{2}\right) \text { : } \\
\text { Ventilación no invasiva o } \mathrm{CPAP} \geq 5 \mathrm{cmH}_{2} \mathrm{O} \text { a través de una } \\
\text { máscara facial completa } \\
\text { - } \mathrm{PaO}_{2} / \mathrm{FiO}_{2} \leq 300 \mathrm{mmHg} \text { o } \mathrm{SpO}_{2} / \mathrm{FiO}_{2} \leq 264 \\
\text { Ventilación invasiva: } \\
\text { - SDRA leve: } 4 \leq \mathrm{OI}<8 \text { o } 5 \leq \mathrm{OSI}<7.5 \\
\text { - SDRA moderado: } 8 \leq \mathrm{Ol}<1607.5 \leq \mathrm{OSI}<12.3 \\
\text { - SDRA grave: } \mathrm{OI} \geq 160 \mathrm{OSI}<12.3\end{array}$ & Evaluar el inicio de anticoagulación \\
\hline
\end{tabular}

$\mathrm{BH}=$ biometría hemática; $\mathrm{PCR}=$ proteína $\mathrm{C}$ reactiva; $\mathrm{PCT}$ = procalcitonina; $\mathrm{IV}=$ intravenosos; $\mathrm{TAC}$ = tomografía axial computarizada.

sin embargo, se requieren más estudios al respecto. ${ }^{28,29}$ Es posible que la excreción viral pudiera ser más prolongada en estos pacientes, lo cual tendría implicaciones para su manejo y tratamiento de la enfermedad de base. ${ }^{30}$
Los casos con un evento de fiebre y neutropenia aún sin otra sintomatología que sugiera infección por SARS-CoV-2 deberán incluir una prueba de RT-PCR en exudado nasofaríngeo, además del tratamiento antibacteriano empírico de acuerdo con 
las guías de manejo de neutropenia febril. ${ }^{31}$ En un primer reporte de la experiencia en México en niños con cáncer en un hospital pediátrico de tercer nivel, se encontró infección en $53.3 \%$ de los pacientes con fiebre y neutropenia. ${ }^{24}$

\section{DIAGNÓSTICO DE COVID-19 POR ESTUDIOS DE IMAGEN}

El papel de los estudios de imagen en el diagnóstico de COVID-19 sigue siendo controversial. El Colegio Americano de Radiología ha establecido que tanto las radiografías como las tomografías de tórax no deben utilizarse como método diagnóstico de $\mathrm{CO}$ VID-19. La decisión de realizar cualquier estudio de imagen debe individualizarse y establecer el riesgo-beneficio.

En casos moderados y graves debe considerarse la realización de tomografía de tórax. Los hallazgos varían de acuerdo con la etapa de la enfermedad, enfermedades subyacentes y tratamiento recibido, los más frecuentes son: lesiones subpleurales $>3$ en número, en parches, nodulares, de aspecto en panal de abeja, con densidad variable con patrón en vidrio despulido, consolidación y engrosamiento pleural. Otros signos mucho menos frecuentes son: broncograma aéreo, derrame pleural y crecimiento de ganglios linfáticos mediastinales. ${ }^{32}$

\section{DIAGNÓSTICO DE COVID-19 POR LABORATORIO}

Después de haber identificado un caso como sospechoso de COVID-19, se debe confirmar el diagnóstico por laboratorio mediante toma de muestra; deberá realizarse por el personal designado, quienes deben portar un equipo de protección personal completo (respirador N95 o N100, lentes con protección lateral, bata desechable de manga larga, doble par de guantes, zapatos de seguridad o cubrezapatos).

Pruebas de amplificación de ácidos nucleicos. La prueba de reacción en cadena de polimerasa en tiempo real (RT-PCR) se considera el estándar diagnóstico para la detección de la infección por SARS-CoV-2. Si una persona se expone a un individuo con diagnóstico confirmado, puede existir un periodo de ventana de aproximadamente cinco días entre dicha exposición y la detección de ácidos nucleicos en la prueba.

Se enviará una muestra de exudado faríngeo y otra de exudado nasofaríngeo, las cuales deben colocarse en un mismo tubo con medio de transporte viral. Otras muestras útiles son el lavado broncoalveolar o aspirado traqueal en pacientes hospitalizados de acuerdo con sus condiciones. ${ }^{25}$

Prueba antigénica para SARS-CoV-2. La prueba antigénica rápida para SARS-CoV-2 detecta antígenos virales y debe realizarse únicamente durante los primeros siete días a partir del inicio de los síntomas, no está indicada en personas asintomáticas. Comparada con la RT-PCR es menos sensible (detección de casos positivos), pero con especificidad (detección de casos negativos) similar. Tiene como ventaja que los resultados son inmediatos (15-30 $\min )$.

Serología para SARS-CoV-2. Las pruebas serológicas que detectan anticuerpos contra SARS-CoV-2 también pueden ayudar tanto al diagnóstico de la enfermedad como para medir la respuesta a la vacunación. Sin embargo, la detección de anticuerpos no siempre traduce la existencia de inmunidad protectora, ya que no todos los anticuerpos producidos en respuesta a una infección son neutralizantes. Los anticuerpos IgM son detectables en los primeros cinco días de la infección, mientras que los anticuerpos tipo IgG se observan aproximadamente a los 14 días de la infección y pueden aparecer incluso hasta los 21 días. Estas pruebas no están recomendadas por sí solas para el diagnóstico de infección por SARS-CoV-2. ${ }^{33-35}$

\section{SÍNDROME INFLAMATORIO MULTISISTÉMICO PEDIÁTRICO (PIMS-TS: PEDIATRIC INFLAMMATORY MULTISYSTEM SYNDROME TEMPORALLY ASSOCIATED WITH SARS-COV-2)}

En un número reducido de casos se ha demostrado la existencia de manifestaciones clínicas de hiperinfla-

Tabla 3: Procedimientos generadores de aerosoles.

- Aspiración de secreciones respiratorias

- Inhaloterapia (se recomienda emplear cámara espaciadora)

- Toma de muestras respiratorias

- Oxigenoterapia de alto flujo

- Ventilación no invasiva

- Ventilación manual con mascarilla y bolsa autoinflable (utilizar filtro de alta eficiencia para reducir riesgo)

- Intubación/extubación endotraqueal

- Ventilación mecánica

- Reanimación cardiopulmonar (contar con equipo de protección completo previo al inicio de la reanimación) 
Tabla 4: Criterios para el diagnóstico de síndrome inflamatorio multisistémico pediátrico.

\begin{tabular}{|c|c|}
\hline Organización & Niños y adolescentes < 19 años con: \\
\hline Mundial de la Salud & $\begin{array}{l}\text { - Fiebre > } 3 \text { días de duración más dos de los siguientes: } \\
\text { - Exantema, conjuntivitis bilateral, alteraciones mucocutáneas (oral, pies y manos) } \\
\text { - Hipotensión o choque } \\
\text { - Alteraciones gastrointestinales (diarrea, vómito o dolor) } \\
\text { - Datos de disfunción miocárdica, pericarditis, valvulitis, alteraciones coronarias o elevación de troponina, } \\
\text { péptido natriurético cerebral } \\
\text { - Evidencia de coagulopatía (trombocitopenia, dímero D elevado) } \\
\text { - Elevación de reactantes de fase aguda (proteína C reactiva; VSG y procalcitonina) } \\
\text { Sin otra causa infecciosa que lo explique }\end{array}$ \\
\hline Centros para el Control y & Menores de 21 años con: \\
\hline $\begin{array}{l}\text { Prevención de Enfermedades } \\
\text { (CDC- EUA) }\end{array}$ & $\begin{array}{l}\text { - Fiebre cuantificada de } 38{ }^{\circ} \mathrm{C} \text { o subjetiva de al menos } 24 \text { horas de duración } \\
\text { - Enfermedad severa con compromiso multisistémico (cardiaco, renal, respiratorio, hematológico, gas- } \\
\text { trointestinal y neurológico) } \\
\text { - Evidencia de inflamación: PCR, VSG, fibrinógeno, procalcitonina, DHL elevada, IL-6, linfopenia e hipoal- } \\
\text { buminemia) } \\
\text { Sin otra causa que lo explique } \\
\text { Incluir pacientes con criterios de enfermedad de Kawasaki }\end{array}$ \\
\hline $\begin{array}{l}\text { Royal College of Pediatrics and } \\
\text { Child Health (Reino Unido) }\end{array}$ & $\begin{array}{l}\text { Cualquier niño con: } \\
\text { - Fiebre persistente } \\
\text { - Evidencia de afección multisistémica } \\
\text { Sin otra causa infecciosa que lo explique } \\
\text { Incluir pacientes con criterios de enfermedad de Kawasaki }\end{array}$ \\
\hline
\end{tabular}

VSG = velocidad de sedimentación globular; $\mathrm{PCR}=$ proteína $\mathrm{C}$ reactiva; $\mathrm{DHL}$ = lactato deshidrogenasa.

Modificado de: Nijman RG et al. ${ }^{40}$

mación asociadas con la infección por SARS-CoV-2 parecidas a una enfermedad de Kawasaki, que han sido denominadas como síndrome inflamatorio multisistémico pediátrico, con un espectro de signos y síntomas de severidad variable. ${ }^{36}$ Uno de los primeros reportes en Londres describió ocho pacientes con fiebre, síntomas gastrointestinales y datos de choque, cinco de ellos presentaron conjuntivitis y cuatro tuvieron exantema sin documentarse síntomas respiratorios en ninguno de ellos. Un estudio realizado por Wittaker y colaboradores reportó 58 casos, en los que la media de edad fue de nueve años (rango intercuartílico [RIC)] 5.7-14), 34\% fueron mujeres. Todos los casos presentaron fiebre y síntomas inespecíficos que incluían vómito 36/58 (45\%), dolor abdominal 31/58 (53\%) y diarrea $30 / 58$ (52\%). El exantema estuvo presente en $30 / 58(52 \%)$ e inyección conjuntival en 26/58 (45\%). Al comparar casos de PIMS-TS con casos de enfermedad de Kawasaki se observaron diferencias en los exámenes de laboratorio y que la edad fue mayor en PIMS-TS (media de nueve años [RIC 5.7-14] vs 2.7 años [RIC 1.4-4.7]). ${ }^{37}$ Kaushik y su grupo reportaron que $45 \%$ de 33 pacientes con síndrome inflamatorio multisistémico presentaba comorbilidades como asma $(15 \%)$, rinitis alérgica (9\%), obesidad (6\%) y enfermedad hematológica (6\%). ${ }^{38}$

La patogénesis de la enfermedad es hasta el momento poco clara, existe una hipótesis acerca de una replicación viral diseminada sustentada por la detección de ARN de SARS-CoV-2 y partículas virales en biopsias de tejido. Asimismo, hay poca información acerca de los factores de riesgo, patogénesis, curso clínico y tratamiento, por lo cual se sugiere que el manejo sea multidisciplinario, incluyendo especialistas pediátricos en terapia intensiva, infectología, hematología y reumatología. ${ }^{39}$ Los signos y síntomas que constituyen los criterios diagnósticos para esta entidad se enlistan en la Tabla 4.

\section{DATOS DE LABORATORIO DE PIMS-TS Y ESTUDIOS DE IMAGEN}

Se ha observado que, en el cuadro agudo, en algunos pacientes existe una prueba positiva para SARS-CoV-2 por RT-PCR, pero en la mayoría se documenta exposición previa mediante anticuerpos IgG e IgM positivos. 
En pocos casos no se encuentran antecedentes de exposición ni de infección previa, pudiendo traducir que el cuadro clínico también puede presentarse como postinfeccioso, lo cual deberá considerarse al momento de realizar el diagnóstico con atención a la clínica y signos de alarma. Por lo anterior, el abordaje deberá incluir marcadores de inflamación, evaluación para afección miocárdica e hipercoagulabilidad; asimismo, deberán descartarse otras causas infecciosas mediante toma de hemocultivos, urocultivo, detección de otros virus respiratorios por PCR, carga viral para virus Epstein-Barr, citomegalovirus, adenovirus, parvovirus, enterovirus, Echovirus y VIH. Se solicitará radiografía de tórax, electrocardiograma, ecocardiograma, mientras que el ultrasonido abdominal será en caso de presentar síntomas abdominales.

\section{TRATAMIENTO DE PIMS-TS}

Es esencial que se identifique al paciente con el posible diagnóstico de PIMS para realizar las acciones inmediatas. El reconocimiento temprano, la resucitación (de acuerdo con la gravedad del caso), estabilización y referencia oportuna tienen implicaciones en el desenlace. ${ }^{39,40}$

De acuerdo con el consenso realizado en el Reino Unido se propuso la siguiente clasificación para el diagnóstico y manejo:

PIMS-TS con manifestaciones de enfermedad tipo Kawasaki (completo o incompleto)

1. Primera línea: inmunoglobulina humana 2 gramos/ $\mathrm{kg}$ (en una sola dosis o dividida).

2. Segunda línea: inmunoglobulina humana + metilprednisolona $10-30 \mathrm{mg} / \mathrm{kg} /$ día.

3. Tercera línea: terapia biológica tocilizumab, considerar bajo el contexto de un ensayo clínico controlado (ECC).

Además, se recomienda iniciar tratamiento antiagregante plaquetario de acuerdo con las guías para enfermedad de Kawasaki y continuarlo durante seis semanas.

\section{PIMS-TS con manifestaciones inespecíficas}

1. Individualizar, decidir vigilancia o inicio de tratamiento de acuerdo con criterios de gravedad.

2. Primera línea: inmunoglobulina humana $2 \mathrm{~g} / \mathrm{kg}$ (en una sola dosis o dividida).

3. Segunda línea: segunda dosis de inmunoglobulina humana.

4. Metilprednisolona $10 \mathrm{mg} / \mathrm{kg} / \mathrm{día}{ }^{40}$

\section{TRATAMIENTO DE COVID-19 EN PEDIATRÍA}

Hasta el momento no existe tratamiento que sea seguro y eficaz para eliminar el SARS-CoV-2 que haya sido aprobado para la población pediátrica; en contraparte, algunos fármacos antivirales para pacientes adultos se han autorizado, basados en resultados de ECC que han demostrado ciertas ventajas en comparación con placebo. Por lo anterior, en niños el tratamiento deberá ser individualizado y establecerse de acuerdo con los distintos escenarios clínicos, grupo de edad, padecimiento actual y comorbilidades del paciente.

En general, a falta de un tratamiento específico, el manejo debe estar dirigido a mantener la estabilidad hemodinámica y ventilatoria del paciente con las medidas de soporte necesarias. El uso inadecuado de antibióticos debe evitarse. Si el paciente se presenta aún en temporada de circulación del virus de la influenza, se iniciará oseltamivir a las dosis habituales por cinco días.

La procalcitonina es un indicador de infección bacteriana agregada. La proteína $\mathrm{C}$ reactiva se ha reportado incrementada en los casos con neumonía grave por SARS-CoV-2 y resulta de menor utilidad como biomarcador de infección bacteriana. Si hay leucocitosis, neutrofilia, procalcitonina elevada y una imagen sugestiva en la radiografía de tórax, se iniciará tratamiento recomendado para neumonía comunitaria (cefotaxima $150 \mathrm{mg} / \mathrm{kg} /$ día o cefuroxima $150 \mathrm{mg} / \mathrm{kg} /$ día dividido cada ocho horas IV).

Si el paciente requiere manejo en la Unidad de Cuidados Intensivos, se indicará cefotaxima a $150 \mathrm{mg} /$ $\mathrm{kg} /$ día cada ocho horas IV más vancomicina $60 \mathrm{mg} / \mathrm{kg} /$ IV cada ocho horas. El tratamiento está dirigido a los microorganismos que causan con mayor frecuencia neumonía en pacientes pediátricos: Streptococcus pneumoniae, Staphylococcus aureus y Haemophilus influenzae tipo b en niños no vacunados.

Como se comentó, debido a que hasta el momento no existe evidencia suficiente basada en ECC, no es posible recomendar algún tratamiento específico. A continuación, se presentan los fármacos que han sido evaluados en estudios de investigación a lo largo de la pandemia, tanto antimicrobianos como inmunomoduladores.

\section{TRATAMIENTOS ANTIVIRALES}

\section{Fármacos aprobados en adultos}

Remdesivir: en estudios in vitro, el remdesivir ejerce una potente actividad antiviral contra SARS-CoV-2. Es 
de amplio espectro, análogo nucleósido y una prodroga que actúa como un inhibidor del ARN polimerasa dependiente de ARN. Su mecanismo de acción consiste en competir con el adenosín trifosfato por la incorporación de las cadenas nacientes de ARN viral y, al no causar terminación inmediata de la cadena, el medicamento parece evadir la corrección por exoribonucleasa viral.

Fue aprobado como tratamiento para COVID-19 en algunos países del mundo; en EUA la FDA (por sus siglas en inglés, Food and Drug Administration) lo aprobó para su uso en adultos y pacientes pediátricos hospitalizados mayores de 12 años y con peso mayor a $40 \mathrm{~kg}$. Adicionalmente, se aprobó su uso de emergencia para pacientes con peso mayor o igual a $3.5 \mathrm{~kg}$. Las dosis recomendadas para pacientes hospitalizados pediátricos con peso $<40 \mathrm{~kg}$ son de $5 \mathrm{mg} / \mathrm{kg}$ inicial y de 2.5 $\mathrm{mg} / \mathrm{kg}$ a partir del día dos, con duración de cinco hasta 10 días en pacientes que reciben ventilación mecánica.

De los ensayos clínicos controlados aleatorizados (ECA) realizados, destaca el de Beigel y colaboradores, en el que se incluyeron a 1,062 casos mayores de 18 años con infección por SARS-CoV-2 hospitalizados con alteraciones radiográficas, requerimiento de oxígeno suplementario, ventilación mecánica o ECMO; se observó que el tratamiento con remdesivir redujo significativamente el tiempo de recuperación de los pacientes que requerían oxígeno suplementario, de 10 días en promedio comparada con 15 días en pacientes con placebo con una tasa de recuperación de 1.29, 95\% IC 1.12-1.40, p < 0.001; sin embargo, no hubo diferencia significativa en pacientes con oxígeno de alto flujo, ventilación mecánica o ECMO, concluyendo que no se encontró beneficio alguno en pacientes con COVID-19 moderada a grave. ${ }^{41}$

Por otro lado, en un ECA multinacional con 402 pacientes (adolescentes mayores de 12 años y adultos) hospitalizados con COVID-19, confirmado por laboratorio y con evidencia radiográfica de infiltrados pulmonares, se comparó la efectividad de cinco o 10 días de tratamiento. Los resultados mostraron que se logró mejoría clínica de manera similar en los grupos (10 y 11 días, respectivamente). Los efectos adversos graves fueron más comunes en el grupo de 10 días de tratamiento. Se concluye que no hay diferencia en casos que no reciben ventilación mecánica o ECMO, con duración de cinco o 10 días; sin embargo, dado que en este estudio no tiene un grupo control con placebo, es difícil establecer un beneficio clínico del uso de remdesivir. ${ }^{42}$ En la actualidad, se realiza un ECC en fase 2 en menores de 12 años (CARAVAN study, ClinicalTrials. gov: NCT04431453).

\section{Fármacos NO aprobados para el tratamiento de COVID-19}

Ivermectina: es un fármaco antiparasitario utilizado en el tratamiento de enfermedades como helmintiasis, oncocercosis y escabiosis con un excelente perfil de seguridad. ${ }^{43} \mathrm{El}$ mecanismo de acción propuesto para el tratamiento de COVID-19 es a través de la inhibición de las proteínas alfa/beta-1 nucleares de transporte del huésped, que forman parte del proceso de transporte intracelular que el virus utiliza para infectar al huésped. ${ }^{44}$ Algunos estudios de farmacocinética y farmacodinamia sugieren que para alcanzar concentraciones en plasma necesarias para una eficacia antiviral, se requerirían dosis hasta 100 veces mayores a las aprobadas para el uso en humanos. ${ }^{45,46}$ No obstante, el fármaco parece acumularse en el tejido pulmonar y las concentraciones en plasma son mucho menores a 2 microgramos (la mitad de la concentración máxima inhibitoria contra SARS-CoV-2) in vitro. ${ }^{47}$

En las guías de tratamiento para COVID-19 de los institutos nacionales de salud de los EUA se publicó una postura el 14 de enero de 2021 después de la revisión de los datos clínicos de los estudios publicados que utilizaron ivermectina para el tratamiento. Se concluyó que en la mayoría de los estudios el número de pacientes fue reducido, que se emplearon diferentes esquemas y dosis, los ensayos clínicos fueron de etiqueta abierta (es decir, no cegados) y, en general, los casos también recibían otros medicamentos (doxiciclina, hidroxicloroquina, azitromicina, zinc y corticosteroides), por lo que la verdadera eficacia era muy difícil de evaluar. Tampoco estaba clara la gravedad de la enfermedad y los desenlaces. Por lo anterior, se concluyó que hay datos insuficientes para hacer una recomendación a favor o en contra del uso de ivermectina para el tratamiento de COVID-19. Aunque es de señalar que en diversas partes del mundo actualmente se están realizando otros ECA con mejor diseño de investigación. ${ }^{48}$

Hidroxicloroquina o cloroquina: es una droga antimalárica. La hidroxicloroquina es análoga de la cloroquina y es utilizada en el tratamiento de enfermedades autoinmunes como lupus eritematoso sistémico $\mathrm{y}$ artritis reumatoide.

La cloroquina e hidroxicloroquina pueden bloquear la infección viral: inhibición de enzimas requeridas para la replicación viral, interferir con la glicosilación del receptor celular específico de SARS-CoV-2, así como en procesos de ensamblaje, transporte de proteínas virales y liberación del virus. ${ }^{49}$ Otros mecanismos descritos son: la potencial inhibición del receptor de 
la enzima convertidora de angiotensina 2, acidificación de la membrana de célula receptora que impide la adhesión viral y un efecto inmunomodulador en la liberación de citocinas inflamatorias. ${ }^{50}$ En general, la hidroxicloroquina tiene pocos efectos adversos como prolongación del intervalo QT e interacciones medicamentosas comparada con la cloroquina.

La seguridad y eficacia del uso de la hidroxicloroquina sola o asociada con azitromicina han sido evaluadas en múltiples ECA. En el estudio RECOVERY del Reino Unido, un ECA incluyó hidroxicloroquina en uno de los brazos; se incluyeron 7,513 pacientes que recibieron hidroxicloroquina durante nueve días o hasta el egreso; la mortalidad a 28 días no tuvo diferencia estadísticamente significativa con otros pacientes que no la recibieron. Además, aquellos que recibieron el tratamiento tuvieron mayor estancia hospitalaria y fueron más propensos a requerir intubación. ${ }^{51}$ Otro estudio realizado en EUA y Canadá en 423 adultos incluyó a pacientes con menos de cuatro días de síntomas compatibles con COVID-19 o detección de SARS-CoV-2 por laboratorio o alto riesgo por exposición. Aquéllos que recibieron hidroxicloroquina no mostraron diferencia significativa en la mejoría de síntomas al día 14 y tampoco hubo diferencia en la incidencia de hospitalización, por lo cual puede concluirse que no hubo evidencia en la reducción de la severidad de los síntomas en pacientes con COVID-19. ${ }^{52}$

La recomendación actual es no utilizarla en pacientes hospitalizados y en los ambulatorios sólo se recomienda en el contexto de un ECA en pacientes adultos con COVID-19. En niños está únicamente aprobada para el tratamiento de enfermedades reumatológicas y malaria.

Lopinavir/ritonavir: es un inhibidor de proteasas, las proteasas son enzimas clave para el procesamiento de diferentes poliproteínas de los coronavirus $(\mathrm{CoV})$. $\mathrm{Al}$ tomar en cuenta la similitud con SARS-CoV y MERS$\mathrm{CoV}$ se pudo determinar que las concentraciones séricas de lopinavir (LPV) disminuyen la actividad in vitro de SARS-CoV al bloquear un paso posterior al ingreso del virus a la célula huésped e impedir su replicación. Es un potente inhibidor del citocromo P450 3A, por lo que es capaz de aumentar las concentraciones de medicamentos metabolizados por esta enzima, por lo cual existe riesgo de toxicidad.

Cao y colaboradore ${ }^{53}$ realizaron un estudio en 199 pacientes mayores de 18 años que recibieron lopinavir $400 \mathrm{mg} /$ ritonavir $100 \mathrm{mg}$ vía oral por 14 días o tratamiento estándar; no se reportó diferencia estadísticamente significativa en la tasa de mortalidad en el grupo que recibió el fármaco (19.2\%) comparado con el otro grupo (25\%), tampoco hubo diferencia significativa en la duración de estancia hospitalaria ni en el tiempo de aclaramiento del virus de muestras respiratorias.

Por lo anterior, en la actualidad, no se recomienda su uso fuera del contexto de un ECA. Hasta la fecha no existe información sobre la eficacia en su uso para el tratamiento de COVID-19 en pacientes pediátricos.

\section{INMUNOMODULADORES Y HEMODERIVADOS EVALUADOS EN EL TRATAMIENTO DE COVID-19}

Hasta el momento no hay evidencia de algún beneficio en la inmunomodulación en COVID-19 leve a moderado. Sin embargo, en el caso de la enfermedad grave, existen ciertas situaciones en las que el paciente podría beneficiarse con el uso de inmunomodulación. Se ha propuesto su uso en el escenario clínico en que haya evidencia de hiperinflamación (clínicamente o por laboratorio) y de progresión de la enfermedad a un estado grave o crítico, como se muestra en la Tabla 5.39

Corticosteroides. Los pacientes con COVID-19 desarrollan una respuesta sistémica inflamatoria que conduce a lesión pulmonar y disfunción multiorgánica, por lo cual se ha evaluado ampliamente el uso de esteroides.

El estudio RECOVERY es un ECA multicéntrico realizado en pacientes hospitalizados con COVID-19,

\section{Tabla 5: Uso sugerido de inmunomodulación en COVID-19 grave.}

Evidencia de estado de hiperinflamación

- Fiebre sostenida o recurrente

- Hepatomegalia, esplenomegalia o linfadenopatía

- Niveles elevados de ferritina y/o proteína C reactiva

- Hipofibrinogenemia

- Elevación de IL-6 u otras citocinas proinflamatorias

Deterioro progresivo o evidencia o riesgo de falla orgánica

- Alteraciones cardiacas: pro-BNP o troponina elevada, inestabilidad hemodinámica persistente que no responde a apoyo vasopresor, lactato elevado, evidencia de cardiomiopatía por electrocardiograma, arritmias

- Alteraciones respiratorias: $\mathrm{PaO}_{2} / \mathrm{FiO}_{2}$ alterado, aumento de los requerimientos de $\mathrm{O}_{2}$ rápidamente progresivo

- Evidencia de coagulopatía: dímero D elevado, trombocitopenia, TP o TTP prolongado

- Alteración del estado mental

- Disminución del aclaramiento de creatinina 
en el cual se demostró que la mortalidad a 28 días era menor en los pacientes que recibieron dexametasona durante 10 días comparada con los que recibieron únicamente tratamiento de soporte. Estos beneficios fueron observados en los pacientes que recibían ventilación mecánica invasiva u oxígeno suplementario al momento de ser incluidos en el estudio; sin embargo, no hubo beneficio entre quienes no requerían oxígeno suplementario. ${ }^{54} \mathrm{La}$ dosis es de $6 \mathrm{mg}$ al día, VO o IV, durante 10 días o hasta el egreso. En caso de no encontrarse disponible podrá sustituirse por cualquier esteroide tomando en cuenta sus equivalencias, prefiriendo las de acción intermedia (prednisona y metilprednisolona). ${ }^{55}$ Durante su uso se deben vigilar estrechamente los efectos adversos asociados como hiperglicemia, sobreinfección bacteriana, alteraciones psiquiátricas y necrosis avascular. No se recomienda su uso en pacientes no considerados graves.

En cuanto a las indicaciones en niños, su eficacia y seguridad no han sido evaluadas en este grupo de edad. El estudio RECOVERY no incluyó suficientes pacientes pediátricos, además de que la mortalidad es significativamente menor; sin embargo, el uso de dexametasona podría ser benéfico en pacientes que requieren ventilación mecánica, aunque aún no se tiene información al respecto.

Inmunoglobulina humana (no específica vs SARS-CoV-2). A pesar de los efectos ya conocidos de inmunomodulación de la inmunoglobulina, es poco clara la evidencia en el tratamiento para COVID-19, sobre todo en enfermedad leve o moderada. En pacientes pediátricos su uso es ampliamente conocido incluyendo la enfermedad de Kawasaki, considerándose generalmente segura. Ha sido utilizada como tratamiento del PIMS, especialmente en casos con Kawasaki-like. ${ }^{56}$

Plasma obtenido de pacientes convalecientes. Es obtenido de casos recuperados de COVID-19 y ha sido recomendado como tratamiento empírico en enfermedades como ébola, MERS, SARS-CoV, H5N1 y H1N1. Lo anterior podría traducir que la transfusión de plasma podría ayudar a la supresión viral y modificar la respuesta inflamatoria. ${ }^{57}$

Al momento, no hay evidencia suficiente de su utilidad en ECA. Más de 70,000 pacientes recibieron plasma de personas convalecientes en EUA a través de un programa de acceso extendido sin grupo control. Los resultados sugieren que el plasma con elevados títulos de anticuerpos puede ser de mayor beneficio que el plasma con títulos bajos en pacientes no intubados a los que se les administra en las primeras 72 horas del diagnóstico. ${ }^{58}$ Ya que los pacientes no fueron seguidos rigurosamente, es difícil establecer conclusiones, por lo que al momento no se recomienda el uso de plasma convaleciente para los pacientes con COVID-19.

Inhibidores de interleucina-1 (IL-1). No hay datos de ECA sobre el uso de inhibidores de IL-1 para el tratamiento de pacientes con COVID-19. Anakinra es un antagonista del receptor humano recombinante IL-1. Está aprobado por la FDA para tratar la artritis reumatoide y los síndromes periódicos asociados con la criopirina, específicamente la enfermedad inflamatoria multisistémica de inicio neonatal. Aun cuando su uso en niños con condiciones reumatológicas es amplio, no existe información sobre el tratamiento para COVID-19.

Inhibidores de IL-6. La interleucina IL-6 es un pleiotrópico, una citocina proinflamatoria producida por una variedad de tipos de células, incluidos los linfocitos, monocitos y fibroblastos. La infección por SARS-CoV-2 induce una producción de IL-6 dosis dependiente desde las células epiteliales bronquiales.

La IL-6 está involucrada en la activación de células $\mathrm{T}$, inducción de la secreción de inmunoglobulina, iniciación de la síntesis de proteínas de fase aguda hepática y precursor hematopoyético de la proliferación celular y estimulación de la diferenciación. ${ }^{59}$

La inflamación sistémica y falla respiratoria hipóxica asociada con COVID-19 puede estar asociada a un aumento en la liberación de citocinas traducido en los elevados niveles de IL-6 en sangre. En la actualidad, existen dos tipos de inhibidores de IL-6 aprobados: anticuerpos monoclonales antirreceptor de IL-6 (tocilizumab y sarilumab) y los anticuerpos monoclonales anti-IL-6 (siltuximab).

Tocilizumab. Es un anticuerpo monoclonal inhibidor del receptor de interleucina-6. En una revisión retrospectiva de 21 casos en quienes se administró tocilizumab, se demostró aparente mejoría clínica, radiológica, incremento de linfocitos y disminución de los niveles de proteína C reactiva; sin embargo, el estudio no comparó contra un grupo control y no se reportaron los efectos adversos. ${ }^{60}$ En otro estudio prospectivo que incluyó a 63 adultos hospitalizados con COVID-19 grave, y que tuvo como criterios de inclusión a aquéllos con proteína $\mathrm{C}$ reactiva elevada, alteraciones pulmonares, desaturación $<93 \%$ al aire ambiente o $\mathrm{PaO}_{2} / \mathrm{FiO}_{2}<300 \mathrm{mmHg}$, así como ferritina, dímero D y deshidrogenasa láctica elevada, se les administró tocilizumab intravenoso o subcutáneo, y se observó la disminución de niveles de laboratorio y defervescencia. La tasa de mortalidad fue de $11 \%$. No obstante, todos los pacientes recibieron tratamiento con inhibidor de 
proteasas. El autor reportó una asociación entre el uso de tocilizumab y la mortalidad reducida, aunque no hubo un grupo control para compararlo. ${ }^{61}$ Teniendo en cuenta lo anterior y los efectos adversos asociados como perforación gastrointestinal, reacciones a la infusión y hepatotoxicidad, se debe tener precaución en casos con trombocitopenia y neutropenia. Se requiere mayor cantidad de estudios para recomendar su uso, sobre todo en niños. Dada la poca experiencia, no se recomienda el uso de tocilizumab o sarilumab fuera del contexto de un ECA.

\section{ANTICOAGULACIÓN}

Hasta el momento no se han documentado series de casos pediátricos que reporten complicaciones tromboembólicas, por lo que podría concluirse que el riesgo en niños es menor que el observado en adultos.

A pesar de lo anterior, tomando en cuenta un consenso de expertos, se sugiere la administración de heparina de bajo peso molecular subcutánea (enoxaparina) dos veces por semana para profilaxis (si no existen contraindicaciones además de tromboprofilaxis mecánica) para niños hospitalizados con COVID-19 (incluyendo PIMS-TS) con niveles elevados de dímero D y factores de riesgo de trombosis (enfermedad oncológica activa, antecedentes personales o familiares de eventos tromboembólicos, obesidad), disfunción severa de ventrículo izquierdo $(\mathrm{FE}<30 \%)$ o pacientes graves que no pueden deambular.

Para niños con inestabilidad hemodinámica y afección renal, se sugiere heparina no fraccionada en infusión IV como profilaxis. En caso de enfermedad tromboembólica podría iniciarse tratamiento con ajuste de la dosis según los niveles de anti-Xa. En pacientes con profilaxis anticoagulante previa al ingreso, se debe considerar cambiar a dosis de tratamiento anticoagulante. ${ }^{62,63} \mathrm{El}$ tratamiento tiene que ser indicado por un hematólogo pediatra y ser supervisado de manera estrecha.

Un punto por aclarar sobre las recomendaciones señaladas sobre el uso de fármacos, es que debido a que se siguen realizando estudios, esas recomendaciones están sujetas a cambios al tener disponible mayor evidencia.

\section{CRITERIOS DE EGRESO HOSPITALARIO}

A la fecha no existe un consenso que establezca criterios para el egreso hospitalario de un paciente con COVID-19, por lo anterior es importante individualizar el caso tomando en cuenta las patologías de base, cuadro clínico, duración de la excreción del virus, así como los riesgos que conlleva el prolongar la estancia hospitalaria. Por otro lado, es aconsejable orientar a los padres sobre los cuidados a llevar, así como entregar por escrito las indicaciones y normas de aislamiento en caso de que aún lo requiera.

Por lo anterior, se proponen los siguientes criterios para el egreso hospitalario: afebril durante más de 24 horas; sin necesidad de oxígeno por más de 24 horas; mejoría clínica y de laboratorio; ingesta oral adecuada; posibilidad de trasladarse para recibir atención médica urgente en caso de mala evolución; y factibilidad de completar el tratamiento en casa.

\section{MEDIDAS GENERALES PARA LA PREVENCIÓN DE COVID-19}

Al tomar en cuenta los mecanismos de transmisión del virus SARS-CoV-2, es prioritario continuar con las medidas ya establecidas desde el inicio de la pandemia.

Las recomendaciones de la Organización Mundial de la Salud están enfocadas en la prevención de la transmisión por gotas y contacto, y la transmisión aérea para cuando se generan aerosoles. De esta forma, se recomienda reforzar la importancia del uso racional y apropiado de todo el equipo de protección personal (EEP) de los trabajadores de la salud que están en contacto con pacientes con COVID-19. ${ }^{64}$

Las medidas de protección por el personal de salud deberán mantenerse en forma estricta para evitar los contagios en las unidades médicas que atienden a pacientes con COVID-19, éstas son las siguientes:

1. La higiene de manos en los cinco momentos: antes de tener contacto con el paciente, antes de realizar una tarea aséptica, después del contacto con sangre y líquidos corporales, después del contacto con el paciente y después del contacto con el entorno del paciente.

2. El material que se utiliza en cada paciente debe ser de un solo uso; cuando no es material desechable y necesita ser compartido entre pacientes, limpiarlo y desinfectarlo.

3. Los niños y acompañantes que puedan ser considerados como casos sospechosos de COVID-19 deberán atenderse en un área específica, separados de los demás.

4. A su llegada, el menor y su familiar deberán aplicarse alcohol gel y, si es posible, lavarse las manos con agua y jabón. El menor sólo deberá ser acompañado por un familiar. 
5. Si el niño lo tolera, se deberá colocar mascarilla (cubrebocas) quirúrgica a su llegada al triaje. El familiar también deberá colocarse mascarilla.

6. En el caso de niños que no toleren la colocación de una mascarilla, se deben mantener alejados, al menos dos metros del resto de los pacientes.

7. Se evitará que el paciente y su familiar transiten por distintas áreas del hospital confinándose al área designada para su atención. Si el traslado fuera imprescindible, el paciente siempre deberá utilizar mascarilla quirúrgica. El personal que lo traslade llevará una mascarilla quirúrgica y guantes.

8. Mantener idealmente dos metros (un metro mínimo) de separación con otros sujetos en todo momento, aun cuando tengan el mismo diagnóstico.

9. El personal de salud que atenderá el caso será el mínimo indispensable.

10. El EPP completo consiste en bata impermeable desechable no estéril, guantes, respirador N95, y protección ocular con lentes o gafas protectores o careta facial.

11. El EPP completo se utilizará por todo el personal de salud cuando tenga contacto directo con el paciente, se ubique a una distancia a menos de un metro cuando vaya a realizar procedimientos que generen aerosoles y cuando se requiera tomar las muestras respiratorias para el diagnóstico.

12. El personal de higiene y limpieza deberá utilizar el EPP completo, pero sus guantes serán de uso rudo, y utilizará protección ocular cuando exista riesgo de salpicaduras de material biológico o químico (p. ej: durante el lavado de los frascos de aspiración).

13. El paciente se hospitalizará en el área designada para casos con COVID-19; aunque una alternativa puede ser un cuarto individual que deberá contar con baño y la puerta se mantendrá cerrada.

14. El familiar acompañante de un caso confirmado (que se considera un contacto cercano), deberá utilizar mascarilla facial todo el tiempo, y además se colocará bata y guantes si va a tener contacto directo con el paciente o sus secreciones.

\section{Recomendaciones sobre del uso de mascarillas (cubrebocas) en niños}

De acuerdo con las recomendaciones de la OMS en niños son:

1. Uso en mayores de cinco años, explicado por la adquisición de hitos del desarrollo, que permitan el uso correcto de las mismas con la mínima ayuda requerida por un adulto, así como mínima manipulación de la mascarilla por parte del niño.

2. No deben usarla niños con problemas cognitivos o respiratorios.

3. En niños de seis a 11 años deberá evaluarse su capacidad para la colocación correcta de la mascarilla, y la capacidad de supervisión de un adulto.

4. Los niños mayores de 12 años deberán seguir las recomendaciones para adultos.

5. Como consideraciones especiales en niños con retraso en el neurodesarrollo, problemas auditivos o dificultad en el aprendizaje, en los que el uso de mascarillas podría condicionar mayor dificultad en el aprendizaje, se debe evaluar el riesgo y deberán ofrecerse alternativas como caretas o mascarillas transparentes. ${ }^{65}$

\section{VACUNACIÓN}

En la actualidad, se cuenta ya con vacunas aprobadas que han demostrado una eficacia mayor a 90\%. Hasta el momento no hay vacunas aprobadas para la prevención de la infección por SARS-CoV-2 en menores de 16 años. Se está en espera de los resultados de los ECA, los cuales ya se han iniciado en población pediátrica.

\section{CONCLUSIONES}

Aun cuando la infección por SARS-CoV-2 parece tener un curso leve en la mayoría de los casos en pediatría, los casos graves no reconocidos y sin atención oportuna pueden culminar con la muerte. Tomando en cuenta lo anterior, es necesario reconocer que hasta el momento no existe un fármaco específico capaz de contrarrestar la respuesta inflamatoria producida por SARS-CoV-2 en la población en general. Aunque se ha iniciado la vacunación en algunos países, incluido México, las medidas de prevención deberán mantenerse hasta que se logre el control de la pandemia.

\section{REFERENCIAS}

1. Aragón-Nogales R, Vargas-Almanza I, Miranda-Novales MG. COVID-19 por SARS-CoV-2: la nueva emergencia de salud. Rev Mex Pediatr. 2019; 86(6): 213-218. https://dx.doi. org/10.35366/91871

2. Cui J, Li F, Shi ZL. Origin and evolution of pathogenic coronaviruses. Nat Rev Microbiol. 2019; 17(3): 181-192. doi: 10.1038/s41579-018-0118-9.

3. Schoeman D, Fielding BC. Coronavirus envelope protein: current knowledge. Virol J. 2019; 16(1): 69. doi: 10.1186/s12985-0191182-0.

4. Arif TB. The 501.V2 and B.1.1.7 variants of coronavirus disease 2019 (COVID-19): a new time-bomb in the making? Infect Control 
Hosp Epidemiol. 2021; 1-2. doi: 10.1017/ice.2020.1434. Epub ahead of print.

5. Montaño-Luna VE, Pacheco-Rosas DO, Vázquez-Rosales JG, Labra-Zamora MG, Fuentes-Pacheco Y, Sámano-Aviña M et al. Manejo clínico de casos pediátricos de COVID-19. Rev Med IMSS. 2020. Disponible en: http://revistamedica.imss.gob.mx/editorial/ index.php/revista_medica/article/view/3702/3736

6. Cheng VCC, Wong SC, Chen JHK, Yip CCY, Chuang VWM, Tsang OTY et al. Escalating infection control response to the rapidly evolving epidemiology of the coronavirus disease 2019 (COVID-19) due to SARS-CoV-2 in Hong Kong. Infect Control Hosp Epidemiol. 2020; 41(5): 493-498. doi: 10.1017/ice.2020.58.

7. Centers for Disease Control and Prevention. Coronavirus Disease 2019 (COVID-19). Appendices. [Fecha de acceso 4 de enero 2021] Available in: https://www.cdc.gov/coronavirus/2019ncov/php/contact-tracing/contact-tracing-plan/appendix. html\#contact

8. Lauer SA, Grantz KH, Bi Q, Jones FK, Zheng Q, Meredith $\mathrm{HR}$ et al. The incubation period of coronavirus disease 2019 (COVID-19) from publicly reported confirmed cases: estimation and application. Ann Intern Med. 2020; 172(9): 577-582. doi: 10.7326/M20-0504.

9. Johansson MA, Quandelacy TM, Kada S, Prasad PV, Steele M, Brooks JT et al. SARS-CoV-2 transmission from people without COVID-19 symptoms. JAMA Netw Open. 2021; 4(1): e2035057. doi: 10.1001/jamanetworkopen.2020.35057.

10. WHO Coronavirus disease 2019 (COVID-19). Weekly epidemiological update-12 January 2021. [Fecha de acceso 12 de enero de 2021] Available in: https://www.who.int/emergencies/ diseases/novel-coronavirus-2019/situation-reports

11. Comunicado Técnico diario. Nuevo Coronavirus en el mundo (COVID-19). Secretaría de Salud. [Fecha de acceso 15 de enero de 2021] Disponible en: https://www.gob.mx/cms/ uploads/attachment/file/606686/Comunicado_Tecnico_Diario COVID-19_2021.01.15.pdf

12. Secretaría de Salud. Documentos. Datos abiertos bases históricas. Disponible en: https://www.gob.mx/salud/documentos/ datos-abiertos-bases-historicas-direccion-general-deepidemiologia

13. Livingston $E$, Bucher K. Coronavirus disease 2019 (COVID-19) in Italy. JAMA. 2020; 323(14): 1335. doi: 10.1001/jama.2020.4344.

14. Tagarro A, Epalza C, Santos M, Sanz-Santaeufemia FJ, Otheo E, Moraleda $\mathrm{C}$ et al. Screening and severity of coronavirus disease 2019 (COVID-19) in children in Madrid, Spain. JAMA Pediatr. 2020; e201346. doi: 10.1001/jamapediatrics.2020.1346.

15. Williams PCM, Howard-Jones AR, Hsu P, Palasanthiran $\mathrm{P}$, Gray PE, McMullan BJ et al. SARS-CoV-2 in children: spectrum of disease, transmission and immunopathological underpinnings. Pathology. 2020; 52(7): 801-808. doi: 10.1016/j. pathol.2020.08.001.

16. Cai J, Xu J, Lin D, Yang z, Xu L, Qu Z et al. A case series of children with 2019 novel coronavirus infection: clinical and epidemiological features. Clin Infect Dis. 2020; 71(6): 1547-1551. doi: 10.1093/cid/ ciaa198.

17. Dong Y, Mo X, Hu Y, Qi X, Jiang F, Jiang Z et al. Epidemiology of COVID-19 among children in China. Pediatrics. 2020; 145(6): e20200702. doi: 10.1542/peds.2020-0702.

18. Lu X, Zhang L, Du H, Zhang J, Li YY, Zhang W et al. SARS-CoV-2 infection in children. N Engl J Med. 2020; 382(17): 1663-1665. doi: 10.1056/NEJMc2005073.

19. Ludvigsson JF. Systematic review of COVID-19 in children shows milder cases and a better prognosis than adults. Acta Paediatr. 2020; 109(6): 1088-1095. doi: 10.1111/apa.15270.
20. Kimberlin DW, Stagno S. Can SARS-CoV-2 infection be acquired in utero? More definitive evidence is needed. JAMA. 2020; 323(18): 1788-1789. doi: 10.1001/jama.2020.4868.

21. Chen H, Guo J, Wang C, Luo F, Yu X, Zhang W et al. Clinical characteristics and intrauterine vertical transmission potential of COVID-19 infection in nine pregnant women: a retrospective review of medical records. Lancet. 2020; 395(10226): 809-815. doi: 10.1016/S0140-6736(20)30360-3. Epub 2020 Feb 12. Erratum in: Lancet. 2020; 395(10229): 1038.

22. Boulad F, Kamboj M, Bouvier N, Mauguen A, Kung AL. COVID-19 in children with cancer in New York City. JAMA Oncol. 2020; 6(9): 1459-1460. doi: 10.1001/jamaoncol.2020.2028.

23. Balduzzi A, Brivio E, Rovelli A, Rizzari C, Gasperini S, Melzi ML et al. Lessons after the early management of the COVID-19 outbreak in a pediatric transplant and hemato-oncology center embedded within a COVID-19 dedicated hospital in Lombardia, Italy. Estote parati. Bone Marrow Transplant. 2020; 55(10): 1900-1905. doi: 10.1038/s41409-020-0895-4.

24. López-Aguilar E, Cárdenas-Navarrete R, Simental-Toba A, Pacheco-Rosas D, Thomé-Ortiz P, Soto-Pérez G et al. Children with cancer during COVID-19 pandemic: early experience in Mexico. Pediatr Blood Cancer. 2021; 68(2): e28660. doi: 10.1002/ pbc. 28660

25. Dirección General de Epidemiología. Lineamiento estandarizado para la vigilancia epidemiológica y por laboratorio de la enfermedad respiratoria viral. Diciembre de 2020. [Fecha de acceso 4 de enero del 2021] Disponible en: https://coronavirus.gob.mx/wp-content/ uploads/2020/04/Lineamiento_de_vigilancia_epidemiologica_de_ enfermedad respiratoria-_viral.pdf

26. Asociación Española de Pediatría. Documento de manejo clínico del paciente pediátrico con infección por SARS-CoV-2. Noviembre, 2020. [Fecha de acceso 8 de enero de 2021] Disponible en: https://www.aeped.es/sites/default/files/b26-11-_aep-seip-secipseup._documento_de_manejo_clinico_del_paciente_pediatrico. pdf

27. Hrusak O, Kalina T, Wolf J, Balduzzi A, Provenzi M, Rizzari C et al. Flash survey on severe acute respiratory syndrome coronavirus-2 infections in paediatric patients on anticancer treatment. Eur $J$ Cancer. 2020; 132: 11-16. doi: 10.1016/j.ejca.2020.03.021.

28. André N, Rouger-Gaudichon J, Brethon B, Phulpin A, Thébault E, Pertuisel S et al. COVID-19 in pediatric oncology from French pediatric oncology and hematology centers: high risk of severe forms? Pediatr Blood Cancer. 2020; 67(7): e28392. doi: 10.1002/ pbc.28392.

29. de Rojas T, Pérez-Martínez A, Cela E, Baragaño M, Galán V, Mata C et al. COVID-19 infection in children and adolescents with cancer in Madrid. Pediatr Blood Cancer. 2020; 67(7): e28397. doi: $10.1002 /$ pbc.28397.

30. Sullivan M, Bouffet E, Rodriguez-Galindo C, Luna-Fineman S, Khan MS, Kearns $P$ et al. The COVID-19 pandemic: a rapid global response for children with cancer from SIOP, COG, SIOP-E, SIOP-PODC, IPSO, PROS, CCI, and St Jude Global. Pediatr Blood Cancer. 2020; 67(7): e28409. doi: 10.1002/ pbc.28409.

31. National Comprehensive Cancer Network. Infectious disease management and considerations in cancer patients with documented or suspected COVID-19. 2020. [Fecha de acceso 9 de enero del 2021] Disponible en: https://www.nccn.org/COVID-19/ pdf/ COVID Infections.pdf.

32. Foust AM, McAdam AJ, Chu WC, Garcia-Peña P, Phillips GS, Plut $D$ et al. Practical guide for pediatric pulmonologists on imaging management of pediatric patients with COVID-19. Pediatr Pulmonol. 2020; 55(9): 2213-2224. doi: 10.1002/ppul.24870. 
33. Sethuraman N, Jeremiah SS, Ryo A. Interpreting diagnostic tests for SARS-CoV-2. JAMA. 2020; 323(22): 2249-2251. doi: 10.1001/ jama.2020.8259.

34. Guo L, Ren L, Yang S, Xiao M, Chang D, Yang F et al. Early humoral response to diagnose novel coronavirus disease (COVID-19). Clin Infect Dis. 2020; 71(15): 778-785. doi: 10.1093/ $\mathrm{cid} / \mathrm{ciaa} 310$

35. COVID-19 Treatment Guidelines Panel. Coronavirus disease 2019 (COVID-19) treatment guidelines. National Institutes of Health. Available in: https://files.covid19treatmentguidelines.nih. gov/guidelines/covid19treatmentguidelines.pdf

36. Soma VL, Shust GF, Ratner AJ. Multisystem inflammatory syndrome in children. Curr Opin Pediatr. 2021; 33(1): 152-158. doi: 10.1097/MOP.0000000000000974.

37. Whittaker E, Bamford A, Kenny J, Kaforou M, Jones CE, Shah $P$ et al. Clinical characteristics of 58 children with a pediatric inflammatory multisystem syndrome temporally associated with SARS-CoV-2. JAMA. 2020; 324(3): 259-269. doi: 10.1001/ jama.2020.10369.

38. Kaushik S, Aydin SI, Derespina KR, Bansal PB, Kowalsky S, Trachtman R et al. Multisystem inflammatory syndrome in children associated with severe acute respiratory syndrome coronavirus 2 infection (MIS-C): a multi-institutional study from New York City. J Pediatr. 2020; 224: 24-29. doi: 10.1016/j.jpeds.2020.06.045.

39. Dulek DE, Fuhlbrigge RC, Tribble AC, Connelly JA, Loi MM, EI Chebib $\mathrm{H}$ et al. Multidisciplinary guidance regarding the use of immunomodulatory therapies for acute COVID-19 in pediatric patients. J Pediatric Infect Dis Soc. 2020; piaa098. doi: 10.1093/ jpids/piaa098.

40. Nijman RG, De Guchtenaere A, Koletzko B, Ross Russell R, Copley $S$, Titomanlio L et al. Pediatric inflammatory multisystem syndrome: statement by the pediatric section of the European Society for Emergency Medicine and European Academy of Pediatrics. Front Pediatr. 2020; 8: 490. doi: 10.3389/fped.2020.00490.

41. Beigel JH, Tomashek KM, Dodd LE, Mehta AK, Zingman BS, Kalil AC et al. Remdesivir for the treatment of Covid-19 - final report. N Engl J Med. 2020; 383(19): 1813-1826. doi: 10.1056/ NEJMoa2007764.

42. Spinner CD, Gottlieb RL, Criner GJ, Arribas López JR, Cattelan AM, Soriano Viladomiu A et al. Effect of remdesivir vs standard care on clinical status at 11 days in patients with moderate COVID-19: a randomized clinical trial. JAMA. 2020; 324(11): 1048-1057.

43. Omura S, Crump A. Ivermectin: panacea for resource-poor communities? Trends Parasitol. 2014; 30(9): 445-455. doi: 10.1016/j.pt.2014.07.005.

44. Caly L, Druce JD, Catton MG, Jans DA, Wagstaff KM. The FDA-approved drug ivermectin inhibits the replication of SARSCoV-2 in vitro. Antiviral Res. 2020; 178: 104787. doi: 10.1016/j. antiviral.2020.104787.

45. Chaccour C, Hammann F, Ramón-García S, Rabinovich NR. Ivermectin and COVID-19: keeping rigor in times of urgency. Am J Trop Med Hyg. 2020; 102(6): 1156-1157. doi: 10.4269/ ajtmh.20-0271.

46. Guzzo CA, Furtek CI, Porras AG, Chen C, Tipping R, Clineschmidt CM et al. Safety, tolerability, and pharmacokinetics of escalating high doses of ivermectin in healthy adult subjects. J Clin Pharmacol. 2002; 42(10): 1122-1133. doi: 10.1177/009127002401382731.

47. Arshad U, Pertinez H, Box H, Tatham L, Rajoli RKR, Curley P et al. Prioritization of anti-SARS-CoV-2 drug repurposing opportunities based on plasma and target site concentrations derived from their established human pharmacokinetics. Clin Pharmacol Ther. 2020; 108(4): 775-790. doi: 10.1002/cpt.1909.
48. The COVID-19 Treatment Guidelines. Panel's statement on the use of ivermectin for the treatment of COVID-19. [Fecha de acceso enero 19, 2021] Available in: https://www. Covid19treatmentguidelines.nih.gov/statement-on-ivermectin/

49. Wang M, Cao R, Zhang L, Yang X, Liu J, Xu M et al. Remdesivir and chloroquine effectively inhibit the recently emerged novel coronavirus (2019-nCoV) in vitro. Cell Res. 2020; 30(3): 269-271. doi: 10.1038/s41422-020-0282-0.

50. Vincent MJ, Bergeron E, Benjannet S, Erickson BR, Rollin PE, Ksiazek TG et al. Chloroquine is a potent inhibitor of SARS coronavirus infection and spread. Virol J. 2005; 2: 69. doi: 10.1186/1743-422X-2-69.

51. RECOVERY Collaborative Group, Horby P, Mafham M, Linsell L, Bell JL, Staplin N et al. Effect of hydroxychloroquine in hospitalized patients with Covid-19. N Engl J Med. 2020; 383(21): 2030-2040. doi: 10.1056/NEJMoa2022926.

52. Skipper CP, Pastick KA, Engen NW, Bangdiwala AS, Abassi M, Lofgren SM et al. Hydroxychloroquine in non-hospitalized adults with early COVID-19: a randomized trial. Ann Intern Med. 2020; 173(8): 623-631. doi: 10.7326/M20-4207.

53. Cao B, Wang Y, Wen D, Liu W, Wang J, Fan G et al. A trial of lopinavir-ritonavir in adults hospitalized with severe COVID-19. N Engl J Med. 2020; 382(19): 1787-1799. doi: 10.1056/ NEJMoa2001282.

54. RECOVERY Collaborative Group, Horby P, Lim WS, Emberson JR, Mafham M, Bell JL et al. Dexamethasone in hospitalized patients with Covid-19 - preliminary report. N Engl J Med. 2020; NEJMoa2021436. doi: 10.1056/NEJMoa2021436.

55. Czock D, Keller F, Rasche FM, Häussler U. Pharmacokinetics and pharmacodynamics of systemically administered glucocorticoids. Clin Pharmacokinet. 2005; 44(1): 61-98. doi: 10.2165/00003088200544010-00003.

56. Shao Z, Feng Y, Zhong L, Xie Q, Lei M, Liu Z et al. Clinical efficacy of intravenous immunoglobulin therapy in critical ill patients with COVID-19: a multicenter retrospective cohort study. Clin Trans/ Immunology. 2020; 9(10): e1192. doi: 10.1002/cti2.1192.

57. Wang $X$, Guo $X$, Xin Q, Pan $Y$, Hu Y, Li J et al. Neutralizing antibodies responses to SARS-CoV-2 in COVID-19 inpatients and convalescent patients. Clin Infect Dis. 2020; ciaa721. doi: 10.1093/ $\mathrm{cid} / \mathrm{ciaa} 721$.

58. Joyner MJ, Senefeld JW, Klassen SA, Mills JR, Johnson PW, Theel ES et al. Effect of convalescent plasma on mortality among hospitalized patients with COVID-19: initial threemonth experience. medRxiv. 2020; 2020.08.12.20169359. doi: 10.1101/2020.08.12.20169359.

59. Yoshikawa T, Hill T, Li K, Peters CJ, Tseng CT. Severe acute respiratory syndrome (SARS) coronavirus-induced lung epithelial cytokines exacerbate SARS pathogenesis by modulating intrinsic functions of monocyte-derived macrophages and dendritic cells. $J$ Virol. 2009; 83(7): 3039-3048. doi: 10.1128/JVI.01792-08.

60. Xu X, Han M, Li T, Sun W, Wang D, Fu B et al. Effective treatment of severe COVID-19 patients with tocilizumab. Proc Natl Acad Sci U S A. 2020; 117(20): 10970-10975. doi: 10.1073/ pnas.2005615117.

61. Sciascia S, Apra F, Baffa A, Baldovino S, Boaro D, Boero R et al Pilot prospective open, single-arm multicentre study on off-label use of tocilizumab in patients with severe COVID-19. Clin Exp Rheumatol. 2020; 38(3): 529-532.

62. Loi M, Branchford B, Kim J, Self C, Nuss R. COVID-19 anticoagulation recommendations in children. Pediatr Blood Cancer. 2020; 67(9): e28485. doi: 10.1002/pbc.28485.

63. Goldenberg NA, Sochet A, Albisetti M, Biss T, Bonduel M, Jaffray J et al. Pediatric/neonatal hemostasis and thrombosis subcommittee 
of the ISTH SSC. Consensus-based clinical recommendations and research priorities for anticoagulant thromboprophylaxis in children hospitalized for COVID-19-related illness. J Thromb Haemost. 2020; 18(11): 3099-3105. doi: 10.1111/jth.15073.

64. World Health Organization. Transmission of SARS-CoV-2 implications for infection prevention precautions. 2020. [Fecha de acceso 12 de enero del 2021] Available in: https:// www.who.int/publications/i/item/modes-of-transmission-ofvirus-causing-COVID-19-implications-for-ipc-precautionrecommendations.
65. World Health Organization. Advice on the use of masks in the context of COVID-19. 2020. [Fecha de acceso 12 de enero del 2021] Available in: https://apps.who.int/iris/bitstream/ handle/10665/332293/WHO-2019-nCov-IPC_Masks-2020.4-eng. pdf?sequence=1\&isAllowed=y

Conflicto de intereses: Las autoras declaran que no tienen. 